INPLASY

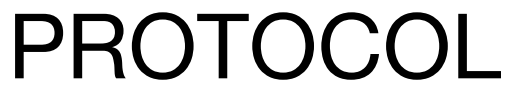

To cite: Liu et al. Efficacy of bimatoprost for the treatment of primary open-angle glaucoma: a protocol of systematic review and metaanalysis. Inplasy protocol 202040118. doi:

10.37766/inplasy2020.4.0118

Received: 19 April 2020

Published: 19 April 2020

Corresponding author: Hong-wei Liu

hongwei200411@163.com

Author Affiliation:

First Affiliated Hospital of Jiamusi University

Support: SRPHLJPHHC (2019-327)

Review Stage at time of this submission: The review has not yet started.

Conflicts of interest: None.

\section{Efficacy of bimatoprost for the treatment of primary open-angle glaucoma: a protocol of systematic review and meta-analysis}

Liu, HW¹; Lu, YT2 Ren, YB³; Meng, Y4.

Review question / Objective: Can bimatoprost effectively treat primary open-angle glaucoma (POAG)?

Condition being studied: Primary open-angle glaucoma and bimatoprost. Information sources: Electronic databases searches. The below electronic database resources will be searched from inception up to the March 1, 2020: MEDLINE, EMBASE, Cumulative Index to Nursing and Allied Health Literature, Cumulative Index to Nursing and Allied Health Literature, Allied and Complementary Medicine Database, Web of Science, Cochrane Library, Chinese Biomedical Literature Database, and China National Knowledge Infrastructure. We will include randomized controlled trials (RCTs) for assessing the efficacy and safety of bimatoprost for the treatment of POAG. The detailed search strategy of MEDLINE is placed. Similar search strategy with specifics for other electronic databases will be presented. Other resources searches: Clinical trials registry, conference/meeting proceedings and reference lists of relevant reviews will be examined to avoid omission.

INPLASY registration number: This protocol was registered with the International Platform of Registered Systematic Review and Meta-Analysis Protocols (INPLASY) on 19 April 2020 and was last updated on 19 April 2020 (registration number INPLASY202040118).

\section{INTRODUCTION}

Review question / Objective: Can bimatoprost effectively treat primary openangle glaucoma (POAG)?

Condition being studied: Primary openangle glaucoma and bimatoprost.

\section{METHODS}

Participant or population: The research patients were definitely diagnosed as POAG, and there will be no restrictions related to the country, age, sex, and other relevant factors. 
Intervention: Studies implemented bimatoprost alone as an experimental treatment regardless its delivery methods, duration, dosage, and frequency.

Comparator: Apart from bimatoprost, there are no restrictions to the control interventions.

Study designs to be included: Only randomized controlled trials (RCTs) that compared efficacy and safety of bimatoprost vs. other treatments for POAG will be included in this study.

Eligibility criteria: Only consider RCTs will be qualified in this research. The literature of animal studies, comments, reviews, case reports, case series, non-RCTs, uncontrolled trials, and quasi-RCTs will not be included.

Information sources: Primary open-angle glaucoma and bimatoprost. Information sources: Electronic databases searches. The below electronic database resources will be searched from inception up to the March 1, 2020: MEDLINE, EMBASE, Cumulative Index to Nursing and Allied Health Literature, Cumulative Index to Nursing and Allied Health Literature, Allied and Complementary Medicine Database, Web of Science, Cochrane Library, Chinese Biomedical Literature Database, and China National Knowledge Infrastructure. We will include randomized controlled trials (RCTs) for assessing the efficacy and safety of bimatoprost for the treatment of POAG. The detailed search strategy of MEDLINE is placed. Similar search strategy with specifics for other electronic databases will be presented. Other resources searches: Clinical trials registry, conference/meeting proceedings and reference lists of relevant reviews will be examined to avoid omission.

Main outcome(s): Primary outcome: 1) Mean intraocular pressure reduction from baseline to the endpoint; 2) Change in best corrected visual acuity. Secondary outcome: 1) Contrast sensitivity; 2) Rate of progression of glaucoma; 3) Quality of life; 4) Incidence of adverse events.
Data management: Two researchers will independently extract data using previous designed standard data extraction sheet. Disagreements regarding data extraction will be settled by consulting a third researcher. The content includes title, first author, year of publication, country, patient characteristics, study design, trial setting, interventions, comparators, outcomes, results, findings, follow-up data, conflict of interests, and other associated information.

Quality assessment / Risk of bias analysis: Two researchers will independently appraise the risk of bias for each eligible trial based on 7 items using The Cochrane Handbook for Systematic Reviews of Interventions Tool. Each item is rated as high, unclear or low risk of bias. Confusion in the interpretation will be solved by a third researcher through discussion.

Strategy of data synthesis: All data will be analyzed using RevMan 5.3 software. All continuous variables will be calculated as mean difference or standardized mean difference and $95 \%$ confidence intervals (CIs). All dichotomous variables will be estimated as risk ratio and $95 \%$ Cls. Chisquare test and 12 statistic will be applied to examine the heterogeneity of eligible trials. $P>0.1$ and/or $12<50 \%$ suggests acceptable heterogeneity, and we will use a fixed-effects model; while $P \leq 0.1$ and/or $12 \geq 50 \%$ indicates obvious significant heterogeneity, and we will utilize a randomeffects model. If acceptable heterogeneity is examined and sufficient data are collected, we will carry out a meta-analysis according to the similarity in study characteristics, interventions, controls, and outcomes. If obvious heterogeneity is tested, we will perform a subgroup analysis to explore the sources of heterogeneity according to the variations in intervention types, research scenario, and outcome tools. In addition, we will also place a sensitivity analysis to test the robustness of study findings by removing trials with high risk of bias. Whenever possible, we will also conduct a funnel plot and Egger's regression test to check reporting bias if over 10 trials are included. 
Subgroup analysis: We will perform a subgroup analysis to explore the sources of heterogeneity according to the variations in intervention types, research scenario, and outcome tools.

Sensibility analysis: We will also place a sensitivity analysis to test the robustness of study findings by removing trials with high risk of bias.

Country(ies) involved: China.

Keywords: Primary open-angle glaucoma; bimatoprost; efficacy; safety. 